\title{
MICROLOCAL HYPO-ANALYTICITY AND HYPO-ANALYTIC PSEUDODIFFERENTIAL OPERATORS
}

\author{
S. BERHANU
}

(Communicated by Walter D. Littman)

ABSTRACT. Let $A$ be a hypo-analytic pseudodifferential operator on a hypoanalytic manifold $\Omega$ of maximal dimension. It is proved here that the operator $A$ decreases the hypo-analytic wave front set of a distribution.

\section{HYPO-ANALYTIC MANIFOLD}

In [1] Baouendi, Chang, and Treves introduced generalizations of real analytic structures which they called hypo-analytic structures. Let $\Omega$ be a $C^{\infty}$ manifold of dimension $m+n$. A hypo-analytic structure on $\Omega$ is the data of an open covering $\left(U_{\alpha}\right)$ of $\Omega$ and for each index $\alpha$, of $m C^{\infty}$ functions $Z_{\alpha}^{1}, \cdots, Z_{\alpha}^{m}$ satisfying the following two conditions:

(i) $d Z_{\alpha}^{1}, \ldots, d Z_{\alpha}^{m}$ are linearly independent at each point of $U_{\alpha}$;

(ii) if $U_{\alpha} \cap U_{\beta} \neq \phi$, there are open neighborhoods $O_{\beta}$ of $Z_{\beta}^{\alpha}\left(U_{\alpha} \cap U_{\beta}\right)$ and $O_{\alpha}$ of $Z_{\alpha}\left(U_{\alpha} \cap U_{\beta}\right)$ and a holomorphic map $F_{\beta}^{\alpha}$ of $O_{\alpha}$ onto $O_{\beta}$, such that

$$
Z_{\beta}=F_{\beta}^{\alpha} \circ Z_{\alpha} \quad \text { on } \quad U_{\alpha} \bigcap U_{\beta} .
$$

We use the notation $Z_{\alpha}=\left(Z_{\alpha}^{1}, \ldots, Z_{\alpha}^{m}\right): U_{\alpha} \rightarrow \mathbb{C}^{m}$. In what follows, we assume that $n=0$ and we refer to $\Omega$ as a hypo-analytic manifold.

Definition 1.1. A distribution $h$ defined on an open neighborhood of a point $p_{0}$ of $\Omega$ is called hypo-analytic at $p_{0}$ if there is a local chart $\left(U_{\alpha}, Z_{\alpha}\right)$ of the above type whose domain contains $p_{0}$ and a holomorphic function $\tilde{h}$ defined of an open neighborhood of $Z_{\alpha}\left(p_{0}\right)$ in $\mathbb{C}^{m}$ such that $h=\tilde{h} \circ Z_{\alpha}$ in a neighborhood of $p_{0}$.

By a hypo-analytic local chart we mean an $m+1$-tuple $\left(U, Z^{1}, \ldots, Z^{m}\right)$ [abbreviated $(U, Z)$ ] consisting of an open subset $U$ of $\Omega$ and of $m$ hypoanalytic functions $Z^{1}, \ldots, Z^{m}$ whose differentials are linearly independent at every point of $U$. We observe that since $\operatorname{dim} \Omega=m$ the mapping $Z=$ $\left(Z^{1}, \ldots, Z^{m}\right): U \rightarrow \mathbb{C}^{m}$ is a local diffeomorphism onto $Z(U)$.

Received by the editors December 1, 1987 and, in revised form, August 17, 1988.

1980 Mathematics Subject Classification (1985 Revision). Primary 35A20; Secondary 35S99. 


\section{STATEMENT OF THE RESULT}

We will reason in a hypo-analytic local chart $(U, Z)$ in $\Omega$. After shrinking $U$ if necessary, we may assume that the mapping $Z=\left(Z^{1}, \ldots, Z^{m}\right): U \rightarrow \mathbb{C}^{m}$ is a diffeomorphism of $U$ onto $Z(U)$ and that $U$ is the domain of local coordinates $x_{j}(1 \leq j \leq m)$ all vanishing at a "central point" which will be denoted by 0 . We will suppose $Z(0)=0$ and denote by $Z_{x}$ the Jacobian matrix of the $Z^{j}$ with respect to the $x_{k}$. Substitution of $Z_{x}(0)^{-1} Z(x)$ for $Z(x)$ will permit us to assume that $Z_{x}(0)=$ the identity matrix. This in turn will allow us to take the real part of the $Z^{j}(j=1, \ldots, m)$ as coordinates and write in these new coordinates $Z^{j}=x_{j}+\sqrt{-1} \phi_{j}(x), j=1, \ldots, m$ where $\phi=\left(\phi_{1}, \ldots, \phi_{m}\right)$ is real-valued whose differential at the origin is 0 . Moreover, the functions $Z^{j}$ are selected so that all the derivatives of order two of the $\phi_{j}$ vanish at the origin. Indeed this can be arranged by replacing each $Z^{j}$ by

$$
Z^{j}-\frac{\sqrt{-1}}{2} \sum \sum \frac{\partial^{2} \phi_{j}}{\partial x_{k} \partial x_{l}}(0) Z^{k} Z^{l}
$$

Such modifications of the $Z^{j}$ preserve hypo-analyticity. Since the derivatives up to order two of $\phi$ vanish at 0 , after contracting $U$ if necessary, we may assume that for all $x, y$ in $U$ :

$$
|\phi(x)-\phi(y)| \leq|x-y| / 2
$$

and

$$
|\phi(y)| \leq \frac{1}{2}|y|^{2}
$$

Defiition 2.1. Let $d$ be a real number. We denote by $\tilde{S}^{d}(U)$ the space of holomorphic functions $\tilde{a}(z, w, \theta)$ in a product set $\mathscr{O} \times \mathscr{O} \times \mathscr{C}$ with $\mathscr{O}$ an open neighborhood of $Z(U)$ and $\mathscr{C}$ an open cone in $\mathbb{C}_{m} \backslash\{0\}$ containing $R_{m} \backslash\{0\}$, which have the following property:

Given any compact subset $K$ of $\mathscr{O}$ and any closed cone $\mathscr{C}^{\prime} \subseteq \mathscr{C}$ whose interior contains $R_{m} \backslash\{0\}$, there is a constant $r>0$ such that for all $z, w$ in $K$ and all $\theta$ in $\mathscr{C}^{\prime}$, we have

$$
|\tilde{a}(z, w, \theta)| \leq r(1+|\theta|)^{d} .
$$

Set $a(x, y, \theta)=\tilde{a}(Z(x), Z(y), \theta)$. For any $\varepsilon>0$ and $u \in C_{c}^{0}(U)$ consider the linear operator $u \rightarrow A^{\varepsilon} u$ defined by

$$
\begin{aligned}
& A^{\varepsilon} u(x) \\
= & \left(\frac{1}{4 \pi^{3}}\right)^{m / 2} \iint \exp \left(\sqrt{-1} \xi \cdot(Z(x)-Z(y))-\varepsilon|\xi|^{2}\right) a(x, y, \xi) u(y) d Z(y) d \xi .
\end{aligned}
$$

Theorems 3.1 and 3.2 of [2] may be consolidated into:

Theorem 2.1. When $\varepsilon \rightarrow 0, A^{\varepsilon}$ converges to a continuous linear operator $A$ : $E^{\prime}(u) \rightarrow D^{\prime}(U)$ which maps $C_{c}^{\infty}(U)$ into $C^{\infty}(U)$ continuously. If $u$ is hypoanalytic at 0 then $A u$ is hypo-analytic at 0.

Following [2] we will call $A$ a hypo-analytic pseudodifferential operator. 
The object of this paper is to prove the following microlocal and hence, more precise version of the above theorem.

Theorem 2.2. Suppose $u \in E^{\prime}(U)$ is hypo-analytic at the covector $\left(0, \xi^{0}\right) \in$ $T^{*} U, \xi^{0} \neq 0$. If $A$ is a hypo-analytic pseudodifferential operator, $A u$ is hypoanalytic at $\left(0, \xi^{0}\right)$.

In [1] the authors microlocalized hypo-analyticity by first adapting Sato's definition [5] and then showing its equivalence with the one derived from the Fourier-Bros-Iagolnitzer transform [4].

\section{Microlocal hYPO-ANAlyticity AND PROOF OF TheOREM 2.2}

Before proceeding to the proof of our theorem it will be convenient to recall Sato's microlocalization as adopted in [1]. They dealt with almost-analytic extensions of the map $Z$. Such an extension is a $C^{\infty}$ mapping $Z_{\#}: U+$ $\sqrt{-1} R_{m} \rightarrow \mathbb{C}^{m}$ such that $Z_{\#}(x)=Z(x)$ for every $x$ in $U$ and $\left(\partial / \partial \bar{z}_{j}\right) Z_{\#}^{k}$ vanishes to infinite order at $\operatorname{Im} z=0$ for all $j, k=1, \ldots, m$. In the sequel $\Gamma$ is a nonempty, acute, and open cone in $R_{m} \backslash\{0\}$. Let $A$ be any open subset of $U, \mathscr{O}$ any open set in $U+\sqrt{-1} R_{m}$ which contains $A$. We shall use the following notation:

$$
N_{\mathscr{O}}(A, \Gamma)=\left\{Z_{\#}(x+\sqrt{-1 v}) \in C^{m}: x \in A, v \in \Gamma \backslash\{0\}, x+\sqrt{-1 v} \in \mathscr{O}\right\},
$$

where $Z_{\#}$ is a given almost-analytic extension of $Z$. We shall denote by $\dot{N}_{\mathscr{O}}(A, \Gamma)$ the interior of the set $N_{\mathscr{O}}(A, \Gamma)$. It is easy to see that there is an open neighborhood $U_{\#}$ of $U$ in $U+\sqrt{-1} R_{m}$ which is mapped diffeomorphically by $Z_{\#}$.

The following lemma and definitions from [1] will be needed.

Lemma 3.1. Let $A$ be an open subset of $U$, and $\mathscr{O}$ an open neighborhood of $A$ in the open set $U_{\#}$ as above. Let $A^{\prime}$ be any relatively compact open subset of $A$, and $\Gamma^{\prime}$ any nonempty open cone in $R_{m} \backslash\{0\}$ whose closure is contained in $\Gamma$. Then there is an open neighborhood $\mathscr{O}^{\prime}$ of $A^{\prime}$ in $U+\sqrt{-1} R_{m}$ such that

$$
\begin{aligned}
& N_{\mathscr{O}^{\prime}}\left(A^{\prime} \cdot \Gamma^{\prime}\right) \subseteq\left\{Z(x)+\sqrt{-1} Z_{x}(x) v: x+\sqrt{-1} v \in(A+\sqrt{-1} \Gamma) \bigcap \mathscr{O}\right\}, \\
& \left\{Z(x)+\sqrt{-1} Z_{x}(x) v: x+\sqrt{-1} v \in\left(A^{\prime}+\sqrt{-1} \Gamma^{\prime}\right) \bigcap \mathscr{O}^{\prime}\right\} \subseteq N_{\mathscr{O}}(A, \Gamma) .
\end{aligned}
$$

Defiition 3.1. We denote by $B_{\mathscr{O}}^{\prime}(A, \Gamma)$ the space of holomorphic functions $\tilde{f}$ in $\dot{N}_{\mathscr{O}}(A, \Gamma)$ which have the following property:

To every compact subset $\tilde{K}$ of $N_{\mathscr{O}}(A, \Gamma)$ there are an integer $k \geq 0$ and a constant $c>0$ such that

$$
|\tilde{f}(z)| \leq c\left(\operatorname{dist}[z, Z(A])^{-k} \quad \text { for all } z\right.
$$

in $\tilde{K} \cap \dot{N}_{\mathscr{\theta}}(A, \Gamma)$.

In what follows the neighborhood $\mathscr{O}$ of $A$ will be such that $Z_{\#}$ maps $(A+$ $\sqrt{-1} \Gamma) \cap \mathscr{O}$ onto $N_{\mathscr{\theta}}(A, \Gamma)$ diffeomorphically.

Let $\tilde{f}$ belong to $B_{\mathscr{O}}^{\prime}(A, \Gamma)$ and set $f=\tilde{f} \circ Z_{\#}$. For $t>0$ and $t$ small, consider

$$
|\sigma|^{-1} \iint_{\sigma} f(x+\sqrt{-1} t \dot{v}) \chi(x) d x d \dot{v}
$$


where $d \dot{v}$ is the measure on $S^{m-1}$ induced by the Lebesgue measure on $R^{m},|\sigma|$ is the measure of the set $\sigma$, and $\chi$ is an arbitrary $C^{\infty}$ function compactly supported in $A$.

Definition 3.2. We define the boundary value $b f$ of $f$ on $A$ by the formula

$$
\int b f(x) \psi(x) d Z(x)=\lim _{t \rightarrow+0}|\sigma|^{-1} \iint_{\sigma} f(x+\sqrt{-1} t \dot{v}) \psi(x) d Z(x) d \dot{v},
$$

where $\psi$ is an arbitrary element of $C_{c}^{\infty}(A)$, and $\sigma$ is a relatively compact measurable subset of $\Gamma \cap S^{m-1}$.

Definition 3.3. Let $u \in \mathscr{D}^{\prime}(U)$ and $(x, \xi)$ be a point in $U \times\left(R_{m} \backslash\{0\}\right)$. We say that $u$ is hypo-analytic at $(x, \xi)$ if there are an open neighborhood $V \subseteq U$ of $x$, an open neighborhood $\mathscr{O}$ of $V$ in $\mathbb{C}^{m}$ and a finite collection of nonempty acute open cones $\Gamma_{k}$ in $R_{m} \backslash\{0\} \quad(k=1, \ldots, \nu)$, such that the following hold:

for every $k=1, \ldots, \nu$ and every $v \in \Gamma_{k},\langle\xi, v\rangle<0$;

for each $k=1, \ldots, \nu$, there is $\tilde{f}_{k} \in B_{\mathscr{O}}^{\prime}\left(V, \Gamma_{k}\right)$ such that in $V$

$$
u=b \tilde{f}_{1}+\cdots+b \tilde{f}_{\nu} .
$$

Definition 3.3 is motivated by the fact that if $u$ is hypo-analytic at $(x, \xi)$ for every $\xi \neq 0$, then $u$ is hypo-analytic at $x$. We will use it in the proof of our theorem.

Proof of Theorem 2.2. We first extend the map $Z$, that is, the map $\phi$, to the whole space $R^{m}$, so that it continues to satisfy (2.1) (this might require a contraction of $U$ ); we may choose our extension so that $\phi$ is compactly supported is some neighborhood of the closure of $U$. We consider the holomorphic functions

$$
\begin{aligned}
& \tilde{A}^{\varepsilon} u(z) \\
= & \left(\frac{1}{4 \pi^{3}}\right)^{m / 2} \iint_{U R_{m}} \exp \left(\sqrt{-1} \xi \cdot(z-Z(y))-\varepsilon|\xi|^{2}\right) a(z, Z(y), \xi) u(y) d Z(y) d \xi .
\end{aligned}
$$

For $\kappa>0$ and $\xi \neq 0$, we have

$$
\int_{R_{m}} \exp \left(-\kappa|\xi|[Z(s)-Z(y)]^{2}\right) d Z(s)=\left(\frac{\pi}{\kappa|\xi|}\right)^{m / 2} .
$$

We may therefore write $\tilde{A}^{\varepsilon} u(z)$ as

$$
\begin{array}{r}
\tilde{A}^{\varepsilon} u(z)=\left(\frac{\kappa}{4 \pi^{4}}\right)^{m / 2} \iiint \exp \left(\sqrt{-1} \xi \cdot(z-Z(y))-\kappa|\xi|(Z(s)-Z(y))^{2}-\varepsilon|\xi|^{2}\right) \\
\cdot a(z, Z(y), \xi) \cdot|\xi|^{m / 2} u(y) d Z(y) d Z(s) d \xi .
\end{array}
$$

The transpose of the inverse of $Z_{x}$ will be denoted by $Z_{x}^{*}$.

We can change contour in the $\xi$-integration from $R_{m}$ to the image of $R_{m}$ under the map $\zeta(\xi)=Z_{x}^{*}(s) \xi+4 \sqrt{-1} B|\xi|(z-Z(y))$, where $B$ is a constant 
satisfying $\left|\operatorname{Im}\left\{Z_{x}(s)^{-1}(Z(x)-Z(s))\right\}\right| \leq B|x-s|^{2}, z$ and $y$ are sufficiently close to 0 . We choose cones $\left(\Gamma_{j}\right)_{0 \leq j \leq \nu}$ satisfying

(i) $R_{m}=\bigcup_{j=0}^{\nu} \Gamma_{j}$,

(ii) measure ${ }_{j \neq k}\left(\Gamma_{j} \cap \Gamma_{k}\right)=0$, and

(iii) $\xi^{0} \notin$ the closed convex hull of $\Gamma_{j}$ for $j=1, \ldots, \nu$.

For each $j=1, \ldots, \nu$ we can find a strictly convex cone $C_{j}$ in $R_{m} \backslash\{0\}$ and a constant $c_{0}>0$ such that $\forall v \in C_{j}$ and $\forall \theta \in \Gamma_{j}, v \cdot \xi^{0}<0$ while $v \cdot \theta \geq c_{0}|v||\theta|$.

For each $j=0,1, \ldots, \nu$ and every $\varepsilon>0$, define

$$
\begin{aligned}
\tilde{J}_{j}^{\varepsilon} u(z)=\left(\frac{\kappa}{4 \pi^{4}}\right)^{m / 2} \iiint_{\xi \in \Gamma_{j}} \exp (\sqrt{-1} \zeta(\xi) \cdot(z-Z(y)) \\
\left.-\kappa\langle\zeta(\xi)\rangle(Z(s)-Z(y))^{2}-\varepsilon\langle\zeta(\xi)\rangle^{2}\right) \\
\cdot a(z, Z(y), \zeta(\xi))\langle\zeta(\xi)\rangle^{m / 2} u(y) \operatorname{det} \frac{\partial \zeta}{\partial \xi} d Z(y) d Z(s) d \xi
\end{aligned}
$$

and let $J_{j}^{\varepsilon} u(x)=\tilde{J}_{j}^{\varepsilon} u(Z(x))$. The proof of Theorem 2.1 in [2] shows that as $\varepsilon \rightarrow+0$ each $J_{j}^{\varepsilon} u$ converges in $\mathscr{D}^{\prime}(U)$ to a distribution which we denote by $J_{j} u$. Moreover, $A u=\sum_{j=0}^{\nu} J_{j} u$.

The theorem will follow from establishing that $\left(0, \xi^{0}\right) \notin W F_{h a}\left(J_{j} u\right), j=$ $0,2, \ldots, \nu$. Consider first $\tilde{J}_{j}^{\varepsilon} u(z)$ for some $j \geq 1$. We will show that the holomorphic functions $\tilde{J}_{j}^{\varepsilon} u(z)$ converge uniformly in a neighborhood of each point $Z(x)+\sqrt{-1} Z_{x}(x) v$ for each $x \in U$ and $v \in C_{j}, v$ small enough. In order to estimate the integrand in $\tilde{J}_{j}^{\varepsilon} u\left(Z(x)+\sqrt{-1} Z_{x}(x) v\right)$ we look at the quantity

$$
\begin{aligned}
\mathscr{Q}=\operatorname{Re} & \left\{\sqrt{-1} Z_{x}^{*}(s) \xi \cdot\left(Z(x)+\sqrt{-1} Z_{x}(x) v-Z(y)\right)-4 B|\xi|(Z(x)\right. \\
& \left.\left.+\sqrt{-1} Z_{x}(x) v-Z(y)\right)^{2}-\kappa\langle\zeta(\xi)\rangle(Z(s)-Z(y))^{2}\right\}
\end{aligned}
$$

where $\zeta(\xi)$ here $=Z_{x}^{*}(s) \xi+4 \sqrt{-1 B}|\xi|\left(Z(x)+\sqrt{-1} Z_{x}(x) v-Z(y)\right)$.

We will estimate each of the three terms in $\mathscr{Q}$ :

$$
\begin{aligned}
\operatorname{Re}\{ & \left.\sqrt{-1} Z_{x}^{*}(s) \xi \cdot\left(Z(x)+\sqrt{-1} Z_{x}(x) v-Z(y)\right)\right\} \\
= & \operatorname{Re}\left\{\sqrt{-1} Z_{x}^{*}(s) \xi \cdot(Z(x)-Z(y))\right\}-\operatorname{Re}\left\{\left(Z_{x}^{*}(s) \xi\right) \cdot\left(Z_{x}(x) v\right)\right\} \\
= & \operatorname{Re}\left\{\sqrt{-1} Z_{x}^{*}(s) \xi \cdot(Z(x)-Z(s))\right\} \\
& +\operatorname{Re}\left\{\sqrt{-1} Z_{x}^{*}(s) \xi \cdot(Z(s)-Z(y))\right\}-\operatorname{Re}\left\{Z_{x}^{*}(s) \xi \cdot Z_{x}(x) v\right\} \\
\leq & B\left(|x-s|^{2}+|s-y|^{2}\right)|\xi|-\operatorname{Re}\left\{\left(Z_{x}^{*}(s) \xi\right) \cdot\left(Z_{x}(x) v\right)\right\} \\
\leq & B\left(|x-s|^{2}+|s-y|^{2}\right)|\xi|-c_{0} / 2|v||\xi| \quad \text { (Since }\left|\phi^{\prime}\right| \text { is small enough.) } \\
\leq & B\left(2|x-y|^{2}+3|s-y|^{2}\right)|\xi|-c_{0} / 2|v||\xi| .
\end{aligned}
$$


In estimating the second term of $\mathscr{Q}$ we will repeatedly use $(2.1)$ and the inequality $|a b| \leq\left(\delta^{2} / 2\right) a^{2}+\left(1 / 2 \delta^{2}\right) b^{2}$.

$$
\begin{aligned}
\operatorname{Re}\{- & \left.4 B|\xi|\left(Z(x)+\sqrt{-1} Z_{x}(x) v-Z(y)\right)^{2}\right\} \\
= & B|\xi|\left\{-\operatorname{Re}(Z(x)-Z(y))^{2}+4 \operatorname{Re}\left(Z_{x}(x) v\right) \cdot\left(Z_{x}(x) v\right)\right. \\
& \left.-8 \operatorname{Re}\left[\sqrt{-1}(Z(x)-Z(y)) \cdot\left(Z_{x}(x) v\right)\right]\right\} \\
\leq & B|\xi|\left\{-3|x-y|^{2}+4|v|^{2}-8 \operatorname{Re}\left[\sqrt{-1}(Z(x)-Z(y)) \cdot\left(Z_{x}(x) v\right)\right]\right\} \\
\leq & B|\xi|\left\{-3|x-y|^{2}+4|v|^{2}+8\left[(x-y) \cdot \phi^{\prime}(x) v+(\phi(x)-\phi(y)) \cdot v\right]\right\} \\
\leq & B|\xi|\left\{-3|x-y|^{2}+4|v|^{2}\right. \\
& \left.+8\left(\frac{\delta^{2}}{2}|x-y|^{2}+\frac{1}{2 \delta^{2}}\left|\phi^{\prime}(x) v\right|^{2}+\frac{\delta^{2}}{2}|\phi(x)-\phi(y)|^{2}+\frac{1}{2 \delta^{2}}|v|^{2}\right)\right\} \\
\leq & B|\xi|\left\{-3|x-y|^{2}+4|v|^{2}\right. \\
& \left.+8\left(\frac{\delta^{2}}{2}|x-y|^{2}+\frac{1}{4 \delta^{2}}|v|^{2}+\frac{\delta^{2}}{8}|x-y|^{2}+\frac{1}{2 \delta^{2}}|v|^{2}\right)\right\} \\
\leq & B|\xi|\left\{\left(-3+5 \delta^{2}\right)|x-y|^{2}+\left(4+\frac{6}{\delta^{2}}\right)|v|^{2}\right\} .
\end{aligned}
$$

For $y, x$, and $v$ sufficiently small, we get:

$$
\operatorname{Re}\left\{-\kappa\langle\zeta(\xi)\rangle(Z(s)-Z(y))^{2}\right\} \leq \frac{-\kappa}{2}|\xi||s-y|^{2}
$$

These estimates add up to give the following estimation on $\mathscr{Q}$ :

$\mathscr{Q} \leq|\xi|\left\{B\left(5 \delta^{2}-1\right)|x-y|^{2}+B\left(4+\frac{6}{\delta^{2}}\right)|v|^{2}+\left(3 B-\frac{\kappa}{2}\right)|s-y|^{2}-\frac{B c_{0}}{2}|v|\right\}$.

We now choose $\delta=1 / \sqrt{10},|v|<(1 / 20) c_{0}$ and $\kappa>8 B$. Then $\mathscr{Q} \leq$ $-B|\xi|\left(\frac{1}{2}|x-y|^{2}+|s-y|^{2}+\left(c_{0} / 4\right)|v|\right)$.

It follows that the absolute value of the integrand in $\tilde{J}_{j}^{\varepsilon} u\left(Z(x)+\sqrt{-1} Z_{x}(x) v\right)$ is dominated by a constant multiple of

$$
(1+|\xi|)^{d+m / 2}|u(y)| e^{-B\left(\frac{1}{2}|x-y|^{2}+|s-y|^{2}+\left(c_{0} / 8\right)|v|\right)|\xi|} .
$$

(Here by using the proof of Theorem 2.1 we are assuming that $u$ is continuous and that the degree $d$ of the amplitude is as small as we like.)

Moreover, if $x^{\prime}$ is close to $x$ and $v^{\prime}$ is near $v$, that is, in a sufficiently small neighborhood of the point $Z(x)+\sqrt{-1} Z_{x}(x) v$, the corresponding integrand is dominated by a constant times

$$
(1+|\xi|)^{d+m / 2}|u(y)| e^{-B\left(\frac{1}{2}|x-y|^{2}+|s-y|^{2}+\left(c_{0} / 8\right)|y|\right)|\xi|} .
$$


It follows that there is $\delta>0$ for which $\left(\tilde{J}_{j}^{\varepsilon} u\right)_{\varepsilon}$ converges locally uniformly on $\left\{Z(x)+\sqrt{-1} Z_{x}(x) v: x \in U, v \in C_{j},|v|<\delta\right\}$ to a holomorphic function $\tilde{J}_{j} u$. Suppose $A^{\prime}$ is a neighborhood of $0, A^{\prime} \subseteq U$ and $C_{j}^{\prime}$ is a cone whose closure is contained in $C_{j}$. By Lemma 3.1 we can find a neighborhood $\mathscr{O}^{\prime}$ of $A^{\prime}$ in $\mathbb{C}^{m}$ such that $\dot{N}_{\mathcal{O}^{\prime}}\left(A^{\prime}, C_{j}^{\prime}\right) \subseteq\left\{Z(x)+\sqrt{-1} Z_{x}(x) v: x \in U, v \in C_{j},|v|<\delta\right\}$.

The estimate we established for $\mathscr{Q}$ implies that $\tilde{J}_{j} u(z)$ belongs to the space $B_{\mathscr{O}^{\prime}}^{\prime}\left(A^{\prime}, C_{j}^{\prime}\right)$. Next fix $v \in C_{j}^{\prime}$. Since the degree of the amplitude $a$ may be assumed to be as small as we wish, the dominated convergence theorem can be applied to conclude that

$$
\lim _{t \rightarrow 0^{+}} \tilde{J}_{j} u\left(Z(x)+\sqrt{-1} Z_{x}(x) t v\right)=J_{j} u(x)
$$

on $x$-compact sets. Therefore, $\left(0, \xi^{0}\right) \notin W F_{h a}\left(J_{j} u\right)$ for $j=1, \ldots, \nu$.

We next consider $\tilde{J}_{0}^{\varepsilon} u(z)$. Since $\left(0, \xi^{0}\right) \notin W F_{h a} u$, in what follows we may assume without loss of generality that $u=b f$ for some $f \in B_{\mathscr{Q}}^{\prime}\left(U, C_{0}\right), \xi^{0} \cdot v<$ 0 for $v \in C_{0}$. We may also assume that the cone $\Gamma_{0}$ was selected so that for some $c_{1}>0, \xi \in \Gamma_{0}$ and $v \in C_{0}, \xi \cdot v \leq-c_{1}|\xi||v|$.

We will write $\tilde{J}_{0}^{\varepsilon} u(z)=\left(\kappa / 4 \pi^{4}\right)^{m / 2} \int_{R_{m}} \int_{\Gamma_{0}} I^{\varepsilon}(z, \xi, s) d Z(s) d \xi$ where

$$
\begin{aligned}
I^{\varepsilon}(z, \xi, s) & \left(\frac{\kappa}{\pi^{4}}\right)^{m / 2} \int_{U} \exp \left(\sqrt{-1} Z_{x}^{*}(s) \xi \cdot(z-Z(y))-4 B|\xi|(z-Z(y))^{2}\right. \\
& \left.-\kappa\langle\zeta(\xi)\rangle(Z(s)-Z(y))^{2}-\varepsilon\langle\zeta(\xi)\rangle^{2}\right) \\
& \cdot\langle\zeta(\xi)\rangle^{m / 2} a(z, Z(y), \zeta(\xi)) g(y) u(y) \operatorname{det} \frac{\partial \zeta}{\partial \xi} d Z(y), \\
\zeta(\xi)= & Z_{x}^{*}(s) \xi+4 \sqrt{-1} B|\xi|(z-Z(y)) \text { and } g \in C_{c}^{\infty}(U), g \equiv 1
\end{aligned}
$$

near the support of $u$. Define

$$
\begin{aligned}
b(z, \omega, \xi, s) & \\
= & a\left(z, \omega, Z_{x}^{*}(s) \xi+4 \sqrt{-1} B|\xi|(z-\omega)\right)\left[\operatorname{det} \frac{\partial}{\partial \xi}\left(Z_{x}^{*}(s) \xi+4 \sqrt{-1} B|\xi|(x-\omega)\right)\right] \\
& \cdot\left\langle Z_{x}^{*}(s) \xi+4 \sqrt{-1} B|\xi|(z-\omega)\right\rangle^{m / 2}
\end{aligned}
$$

and for each $\varepsilon>0$ let

$$
\begin{aligned}
h^{\varepsilon}\left(z^{*}, s\right)=\exp \left\{\sqrt{-1} Z_{x}^{*}(s) \xi \cdot\right. & \left(z-z^{*}\right)-4 B|\xi|\left(z-z^{*}\right)^{2} \\
& \left.-\kappa\langle\zeta\rangle\left(Z(s)-z^{*}\right)^{2}-\varepsilon\langle\zeta\rangle^{2}\right\} \cdot b\left(z, z^{*}, \xi, s\right),
\end{aligned}
$$

where in the latter $\zeta=Z_{x}^{*}(s) \xi+4 \sqrt{-1} B|\xi|\left(z-z^{*}\right)$. 
Recalling that $u=b f$, we have:

$$
\begin{aligned}
I^{\varepsilon}(z, \xi, s)= & \lim _{t \rightarrow+0}\left(\frac{\kappa}{4 \pi^{4}}\right)^{m / 2} \int h^{\varepsilon}(Z(y) \\
& \left.+\sqrt{-1} Z_{y}(y) v\right) f\left(Z(y)+\sqrt{-1} t Z_{y}(y) v\right) g(y) d Z(y) \\
= & \lim _{t \rightarrow+0}^{\left(v \in C_{0}\right)}\left(\frac{\kappa}{4 \pi^{4}}\right)^{m / 2} \int h^{\varepsilon}\left(Z(y)+\sqrt{-1} t Z_{y}(y) v\right) f(Z(y) \\
& \left.+\sqrt{-1} t Z_{y}(y) v\right) g(y) d\left(Z(y)+\sqrt{-1 t} Z_{y}(y) v\right) \\
= & \lim _{t \rightarrow+0}\left(\frac{\kappa}{4 \pi^{4}}\right)^{m / 2} \int_{C_{t}} h^{\varepsilon}\left(z^{*}\right) f\left(z^{*}\right) \tilde{g}\left(z^{*}, t\right) d z^{*}
\end{aligned}
$$

where for $t$ small enough, $\tilde{g}\left(z^{*}, t\right)=g(y)$ and $C_{t}$ is the image of $U$ under the map $y \rightarrow z^{*}=Z(y)+\sqrt{-1} Z_{y}(y) t v$.

Here we may have to shrink $U$ and take $\delta>0$ so that $C_{t}$ is a manifold for $0<t \leq \delta$.

We will apply the techniques employed in the proof of Theorem 2.1 of Chapter II in [1] to establish that for $z$ near $0\left|I^{\varepsilon}(z, \xi, s)\right|$ is uniformly bounded for all $\varepsilon>0$ by an integrable function in $\xi$ and $s$ space. Let $g_{0} \in C_{c}^{\infty}(U), g_{0}=1$ near 0 , supp $g_{0} \subseteq\{x: g(x)=1\}$ and $0 \leq g_{0} \leq 1$. We restrict $t$ to belong to the interval $\left(o, \delta^{\prime}\right), \delta^{\prime}<\delta$ and select $\tilde{s}>0$ such that $\tilde{s}+\delta^{\prime}<\delta$. By Stokes' theorem,

$$
\begin{aligned}
& \int_{C_{t}} h^{\varepsilon}\left(z^{*}\right) f\left(z^{*}\right) g\left(z^{*}, t\right) d z^{*} \\
& =\int_{U} h^{\varepsilon}\left(Z(y)+\sqrt{-1}\left(t+s g_{0}(y)\right) Z_{y}(y) v\right) f\left(Z(y)+\sqrt{-1}\left(t+\tilde{s} g_{0}(y)\right) Z_{y}(y) v\right) \\
& \quad \cdot d\left(Z(y)+\sqrt{-1}\left(t+\tilde{s} g_{0}(y)\right) Z_{y}(y) v\right) .
\end{aligned}
$$

The techniques in [1] that we referred to imply that for all $\varepsilon>0$,

$$
\int_{C_{1}} h^{\varepsilon}\left(z^{*}\right) f\left(z^{*}\right) g\left(z^{*}, t\right) d z^{*} \mid \leq(\text { constant }) \cdot \tilde{\kappa},
$$

where

$$
\begin{gathered}
\tilde{\kappa} \leq \sup \mid \exp \left(\sqrt{-1} Z_{x}(s) \xi \cdot\left(z-z^{* *}\right)-4 B|\xi|\left(z-z^{*}\right)^{2}\right. \\
-\kappa\left\langle Z_{x}(s) \xi+4 \sqrt{-1} B|\xi|\left(z-z^{* *}\right)\right\rangle\left(Z(s)-z^{* *^{2}}\right) \mid \\
z^{* *}=Z(y)+\sqrt{-1}\left(t+\tilde{s} g_{0}(y)\right) Z_{y}(y) v .
\end{gathered}
$$

We note that since the degree of the amplitude, a may be assumed to be as small as we wish, the term $b\left(z, z^{*}, \xi, s\right)$ is bounded. In order to estimate $\tilde{\kappa}$, we look at

$$
\mathscr{Q}(z)=\operatorname{Re}\left\{\left(\sqrt{-1} Z_{x}(s) \xi \cdot\left(z-z^{* *}\right)\right)-4 B|\xi|\left(z-z^{* *}\right)^{2}-\kappa|\xi|\left(Z(s)-z^{* *}\right)^{2}\right\}
$$

which can be compared to

$$
\tilde{\mathscr{Q}}(0)=\operatorname{Re}\left\{-\sqrt{-1} Z_{x}^{*}(s) \xi \cdot z^{* *}-4 B|\xi|\left(z^{* *}\right)^{2}-\kappa|\xi|\left(Z(s)-z^{* *}\right)^{2}\right\}
$$


which in turn is comparable to

$$
\tilde{\mathscr{Q}}=\operatorname{Re}\left\{-\sqrt{-1} \xi \cdot z^{* *}-4 B|\xi|\left(z^{* *}\right)^{2}-\kappa|\xi|\left(Z(s)-z^{* *}\right)^{2}\right\} .
$$

We will use the assumptions that $\phi(0)=0, \phi^{\prime}(0)=0, \phi^{\prime \prime}(0)=0$ and we may also have to shrink the neighborhood $U$. We being by estimating

$$
\begin{aligned}
& \operatorname{Re}\left\{-\sqrt{-1} \xi \cdot z^{* *}-4 B|\xi|\left(z^{* *}\right)^{2}\right\} \\
& =\xi \cdot \phi(y)+\left(t+\tilde{s} g_{0}(y)\right) \xi \cdot v-4 B|\xi|\left(|y|^{2}-|\phi(y)|^{2}\right) \\
& \quad+4 B\left(t+s g_{0}(y)\right)^{2}|\xi| \operatorname{Re}\left(Z_{y}(y) v \cdot Z_{y}(y) v\right) \\
& \quad+8 B|\xi|\left(t+s g_{0}(y)\right)\left[\left(y \cdot \phi^{\prime}(y) v\right)+(\phi(y) \cdot v)\right] \\
& \leq\left(t+\tilde{s} g_{0}(y)\right) \xi \cdot v-c_{2}|y|^{2}|\xi| \quad \text { for some } c_{2}>0 \\
& \leq-c_{1}\left(t+\tilde{s} g_{0}(y)\right)|v||\xi|-c_{2}|y|^{2}|\xi| .
\end{aligned}
$$

Since $g_{0}(y) \equiv 1$ near $y=0$, there is $\tilde{c}_{1}>0$ such that the last quantity $\leq-\left(\tilde{c}_{1}+\left(c_{2} / 2\right)|y|^{2}\right)|\xi|$.

Next we estimate

$$
\begin{aligned}
\operatorname{Re}\left(\left(Z(s)-z^{* *}\right)^{2}\right) & \geq \frac{3}{4}|s-y|^{2}-\left|Z_{y}(y) v\right|^{2}-2|Z(s)-Z(y)|\left|Z_{y}(y) v\right| \\
& \geq \frac{3}{4}|s-y|^{2}-\left|Z_{y}(y) v\right|^{2}-\left(|Z(s)-Z(y)|^{2}\right) / 8-8\left|Z_{y}(y) v\right|^{2} \\
& \geq\left(c_{3} / \kappa\right)|s-y|^{2}-9\left|Z_{y}(y) v\right|^{2} \quad \text { for some } c_{3}>0 .
\end{aligned}
$$

Moreover, for $|v|$ small enough, the latter $\geq\left(c_{3} / \kappa\right)|s-y|^{2}-\tilde{c}_{1} / 2 \kappa$. Combining these estimates we get: $\tilde{\mathscr{Q}} \leq-|\xi|\left(\tilde{c}_{1} / 2+c_{3}|s-y|^{2}+\left(c_{2} / 2\right)|y|^{2}\right)$, which gives the asserted bound on $I^{\varepsilon}(z, \xi, s)$. It follows that $\tilde{J}_{0}^{\varepsilon} u(z)$ converges to a holomorphic function near $z=0$. Hence $\left(0, \xi^{0}\right) \notin W F_{h a}(A u)$.

\section{ACKNOWLEDGMENT}

I wish to express my gratitude to Professor Francois Treves for his advice and encouragement during his direction of my dissertation [3] which is the source of this article.

\section{REFERENCES}

1. M. S. Baouendi, C. H. Chang and F. Treves, Microlocal hypo-analyticity and extensions of $C R$ functions, J. Differential Geom. 18 (1983), 331-391.

2. S. Berhanu, Hypo-analytic pseudodifferential operators Proceedings of Amer. Math. Soc. 105 (1989), 582-588.

3. __, Hypo-analytic pseudodifferential operators, Dissertation, Rutgers University, 1987.

4. J. Bros and D. Iagolnitzer, Support essentiel et structure analytique des distributions, Seminarie Goulaouic-Lions-Schwartz, 1975-76, No. 18.

5. M. Sato, Hyperfunction and partial differential equations, Proc. Int. Conf. Funct. Anal. Tokyo (1969), 91-94.

Department of Mathematics, Temple University, Philadelphia, Pennsylvania 19122 